\title{
Effect of Arteriovenous Fistula on the Cardiovascular Dynamics
}

\author{
Jiro NAKano, M.D.
}

\section{SUMMARY}

The effects of acute A-V fistula of different magnitudes on the cardiovascular dynamics were studied in anesthetized, open-chest dogs. It was found that A-V fistula decreases mean systemic arterial pressure, effective systemic blood flow, total and pulmonary peripheral resistances. On the other hand, A-V fistula increases heart rate, myocardial contractility, total cardiac output, stroke volume, left and right atrial pressures, pulmonary arterial pressure and systemic peripheral resistance. The magnitude of the above hemodynamic changes was essentially in proportion to the size of the fistula. It was also found that $\mathrm{A}-\mathrm{V}$ fistula decreases superior mesenteric and femoral arterial blood flows and increases the peripheral resistances.

\section{Additional Indexing Words :}

Cardiodynamics Myocardial contractility

Systemic circulation

Pulmonary circulation Splanchnic circulation Femoral circulation

Peripheral circulation

$\mathrm{T}$

HE effects of acute and chronic arteriovenous (A-V) fistula on the cardioworkers. ${ }^{1)-6)}$ It has been well established that acute A-V fistula increases heart rate, cardiac output and pulmonary arterial pressure and decreases mean systemic arterial pressure and total peripheral resistance. The present study was undertaken to investigate further the effects of acute A-V fistula on the cardiodynamics, myocardial contractility and peripheral circulations in anesthetized dogs.

\section{METHODS}

Dogs wcighing between 20 and $30 \mathrm{Kg}$. wcre anesthetized with the intravenous administration of $100 \mathrm{mg} . / \mathrm{Kg}$. of a alpha-chloralose or $30 \mathrm{mg} . / \mathrm{Kg}$. of sodium pentobarbital. A variety of experimental techniques and preparations was employed to study the effects of acute A-V fistula on the cardiovascular dynamics. In all experiments, the left hemithorax was opened under artificial respiration. The pericardium was incised and the heart suspended in a pericardial cradle. Blood was rendered incoagulable by the intravenous administration of $5 \mathrm{mg} . / \mathrm{Kg}$. of sodium

From the Departments of Pharmacology and of Medicine, University of Oklahoma School of Medicine, Oklahoma Gity, Oklahoma, U.S.A.

Received for publication March 2, 1971. 
hcparin initially, followed by $2.5 \mathrm{mg}$. $/ \mathrm{Kg}$. every subsequent half hour. In $20 \mathrm{dogs}$, mean systemic arterial pressure, mean pulmonary arterial pressure, left and right atrial pressures were measured continuously with Statham pressure transducers (P23AA and P23D), connected to catheters placed, respectively, in the roots of the aorta and pulmonary artery, and in the left and right atria through a pulmonary vein and branch of the right jugular vein. In $10 \mathrm{dogs}$, left ventricular and atrial pressures were measured continuously with Statham pressure transducers (P23D) connected with a size 15 needle punctured into the left ventricular cavity or with a Statham pressure transducer (SF-1) placed in the left ventricular or atrial cavity through a pulmonary vein. Heart rate and myocardial contractile force were measured continuously using an Electronics for Medicine (EFM) tachometer (Model TDC-1) and a Walton-Brodie strain gauge arch which was sutured directly to the right or left ventricular muscle. Cardiac output (minus coronary blood flow) was measured continuously using a Shipley-Wilson rotameter. The technique employed to measure continuously and simultaneously cardiac output and A-V fistula flow was described previously. ${ }^{4), 6)}$ The A-V fistula consisted of a tygon tube previously filled with arterial blood of donor dogs. The tube was connected between the side arm of the inflow tract of a rotameter and a centrally cannulated external jugular vein. During a control period, the tube was occluded with a screw clamp. A-V fistulas of different magnitudes were produced by unscrewing the clamp progressively. The blood flow of the fistula was measured continuously with a second rotameter. Thus, when the fistula was patent, the total cardiac output (minus coronary blood flow) was equal to the sum of the two measured flow rates, i.e., effective systemic blood flow and fistula flow. ${ }^{5}$ In all dogs, varying sizes of $\mathrm{A}-\mathrm{V}$ fistula were produced suddenly and maintained for 5 to $15 \mathrm{~min}$. In 6 additional dogs, in which the effects of different degrees of $\mathrm{A}-\mathrm{V}$ fistulas on the splanchnic circulation were studied, a mid-line laparotomy was performed; and in addition to the measurements of heart rate, mean systemic arterial pressure, pulmonary arterial pressure, the superior mesenteric arterial pressure, portal venous pressure and the inferior vena caval pressure were measured continuously with Statham pressure transducers (Model P23D). Myocardial contractility was also evaluated by continuous measurement of the maximum rate of the development of the isovolumetric left ventricular pressure (dp/dt) with an EFM R-C differentiator as described previously. ${ }^{7,8)}$ In 6 additional anesthetized dogs, a femoral A-V fistula was produced with a tygon tube, connected between the cannulated femoral artery and a femoral vein. Blood flows in the femoral artery distal to the site of the $A-V$ fistula and in the contralateral femoral artery were measured with 2 Shipley-Wilson rotameters. ${ }^{9}$ In this preparation, bilateral femoral arterial pressures and venous pressures were measured simultaneously and continuously with Statham pressure transducers (P23AA). All of the hemodynamic parameters measured except heart rate were recorded contínuously with an EFM recorder (Model DR8).

Total, systemic, pulmonary and superior mesenteric peripheral resistances were calculated from the following formulas: Total peripheral resistance $(\mathrm{mm} . \mathrm{Hg}$ / $\mathrm{ml} . / \mathrm{min}.)=$ mean systemic arterial pressure $(\mathrm{mm} . \mathrm{Hg}) /$ total cardiac output ( $\mathrm{ml} . / \mathrm{min}$.$) .$ Systemic peripheral resistance $(\mathrm{mm} . \mathrm{Hg} / \mathrm{ml} . / \mathrm{min}$.) = mean systemic arterial pressure (mm.Hg)/effective systemic blood flow ( $\mathrm{ml} . / \mathrm{min}$.). Pulmonary peripheral resistance $(\mathrm{mm} . \mathrm{Hg} / \mathrm{ml} . / \mathrm{min})=.[$ mean , pulmonary arterial pressure $(\mathrm{mm} . \mathrm{Hg})$-mean left atrial pressure (mm.Hg)]/total cardiac output (ml./min.). Superior mesenteric 
peripheral resistance $(\mathrm{mm} . \mathrm{Hg} / \mathrm{ml} . / \mathrm{min})=$. mean systemic arterial pressure $(\mathrm{mm} . \mathrm{Hg})$ - mean portal venous pressure $(\mathrm{mm} . \mathrm{Hg})]$ /superior mesenteric arterial blood flow (ml./min.).

\section{Results}

1. Hemodynamic Changes in the Systemic and Pulmonary Circulations As illustrated in Fig. 1, upon the onset of the A-V fistula, mean systemic arterial pressure and effective systemic blood flow decreased abruptly and markedly, whereas heart rate, mean pulmonary arterial pressure, left and right atrial pressures and myocardial contractility increased significantly. Within approximately 1-2 min. after its onset, all hemodynamic parameters measured, except fistula flow, returned toward control values and became fairly well stabilized at levels significantly below or above controls (Fig. 1). During a period of A-V fistula, total cardiac output (the sum of effective systemic blood flow and fistula flow), stroke volume and systemic peripheral resistance increased considerably, whereas total and pulmonary peripheral resistances decreased below controls. The sudden closure of the A-V fistula increased markedly mean systemic arterial pressure and effective systemic blood flow, but decreased heart rate, pulmonary arterial pressure, left and right atrial pressures, myocardial contractility and systemic peripheral resist-

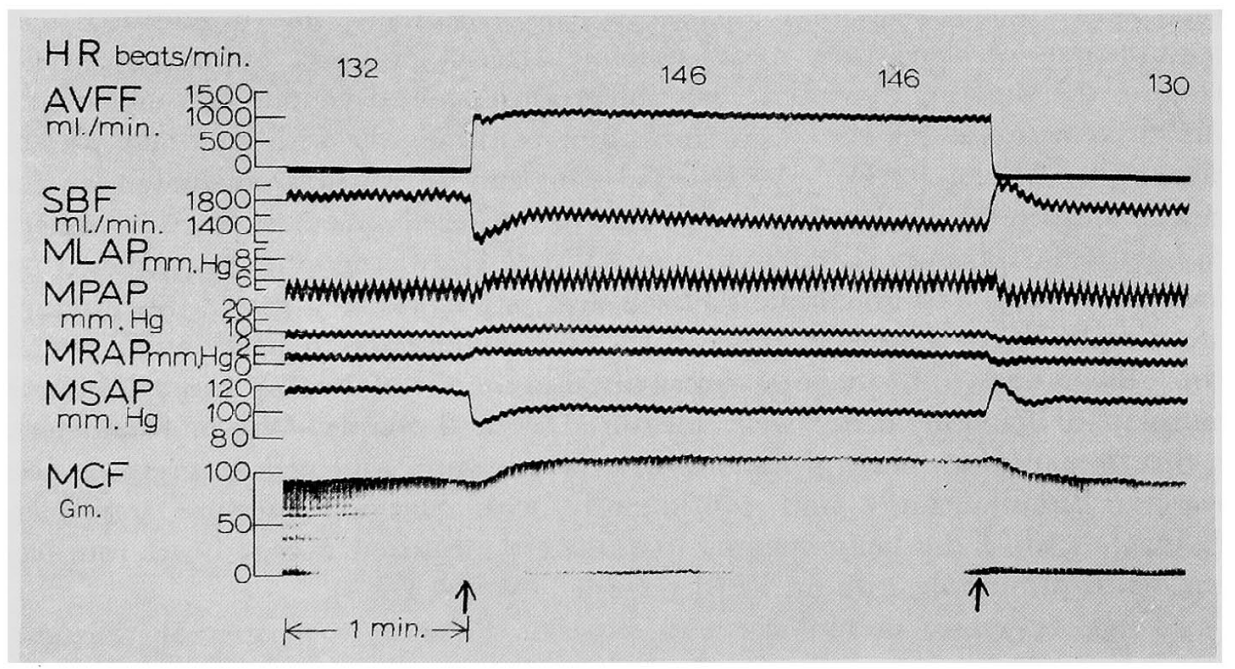

Fig. 1. The effects of a large A-V fistula on heart rate (HR), A-V fistula blood flow (AVFF), effective systemic blood flow (SBF), mean left atrial pressure (MLAP), mean pulmonary arterial pressure (MPAP), mean right atrial pressure (MRAP), mean systemic arterial pressure and myocardial contractile force (MCF) in a dog. A-V fistula was induced between 2 vertical arrows. 


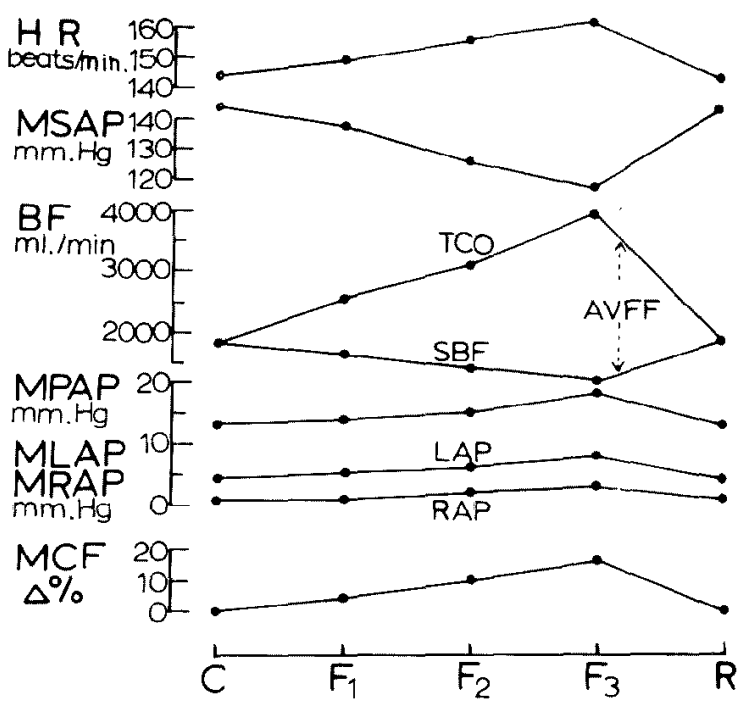

Fig. 2. The effects of stepwise increases in A-V fistula on heart rate (HR), mean systemic arterial blood pressure (MSAP), total cardiac output (TCO), A-V fistula blood flow (AVFF), effective systemic blood flow (SBF), mean pulmonary arterial pressure (MPAP), mean left atrial pressure (MLAP), mean right atrial pressure (MRAP) and myocardial contractile force (MCF) in a dog. $G$ and $R$ denote control and recovery values, respectively. $F_{1-3}$ denote values in A.V fistulas of severity increasing stepwise.

ance. These parameters returned gradually to control values within about 3-4 min. As shown in Fig. 2, the magnitude of the hemodynamic changes is essentially proportional to the severity of the A-V fistula. The magnitude of the decrease in effective systemic flow was much smaller than that of the increase in either total cardiac output or fistula flow. Total peripheral resistance decreased together with the decrease in mean systemic arterial pressure, whereas pulmonary peripheral resistance decreased progressively as mean pulmonary arterial pressure and total pulmonary flow increased. However, when total pulmonary blood flow increased markedly, pulmonary peripheral resistance reached a minimum and remained at that level.

2. Effects of A-V Fistula on the Cardiodynamics and Myocardial Contractility

As seen in Fig. 3, the sudden onset of A-V fistula decreased aortic systolic, diastolic and mean pressures and increased pulse pressure. Within 1 min., aortic pressure returned toward control and stabilized at a level significantly below control. On the other hand, A-V fistula decreased left ventricular and end-diastolic pressure, and increased the right ventricular systolic and enddiastolic pressures and increased $a$ wave of the left atrial pressure. After the 


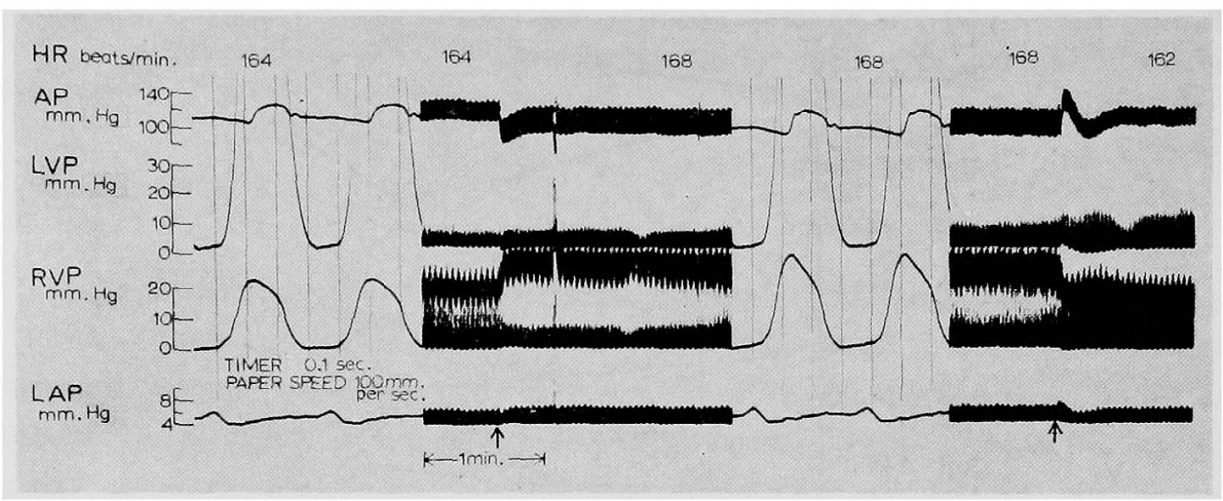

Fig. 3. The effects of A-V fistula on heart rate (HR), aortic pressure (AP), left ventricular pressure (LVP), right ventricular pressure (RVP) and left atrial pressure (LAP) in a dog. A-V fistula was induced between 2 vertical arrows.

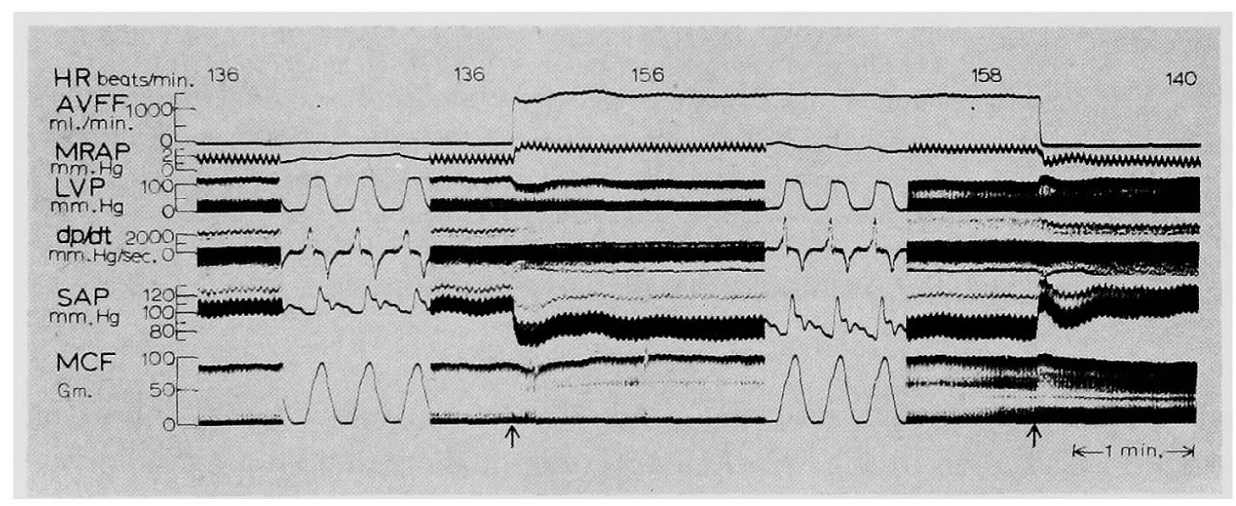

Fig. 4. The effects of A-V fistula on heart rate (HR), A-V fistula blood flow (AVFF), mean right atrial pressure (MRAP), left ventricular pressure (LVP), the maximum rate of the development of the left ventricular isometric tension (dp/dt), systemic arterial pressure (SAP) and myocardial contractile force (MCF) in a $\operatorname{dog}$. A-V fistula was induced between 2 vertical arrows.

initial changes, the left and right end-diastolic pressures returned to or toward control values. Upon cessation of A-V fistula, both left ventricular enddiastolic pressure and left atrial pressure increased very transiently and then decreased to levels below controls before their gradual return to control values within $1 \mathrm{~min}$. On the other hand, right ventricular end-diastolic pressure decreased transiently and slightly, and returned to control within $1 \mathrm{~min}$. Fig. 4 also shows that $\mathrm{A}-\mathrm{V}$ fistula caused qualitatively similar evolutional changes in the right atrial pressure, left ventricular pressure and myocardial contractile force as described previously (Fig. 1 and Fig. 3). In addition, A-V fistula increased the maximum rate of the development of the left ventricular isometric 


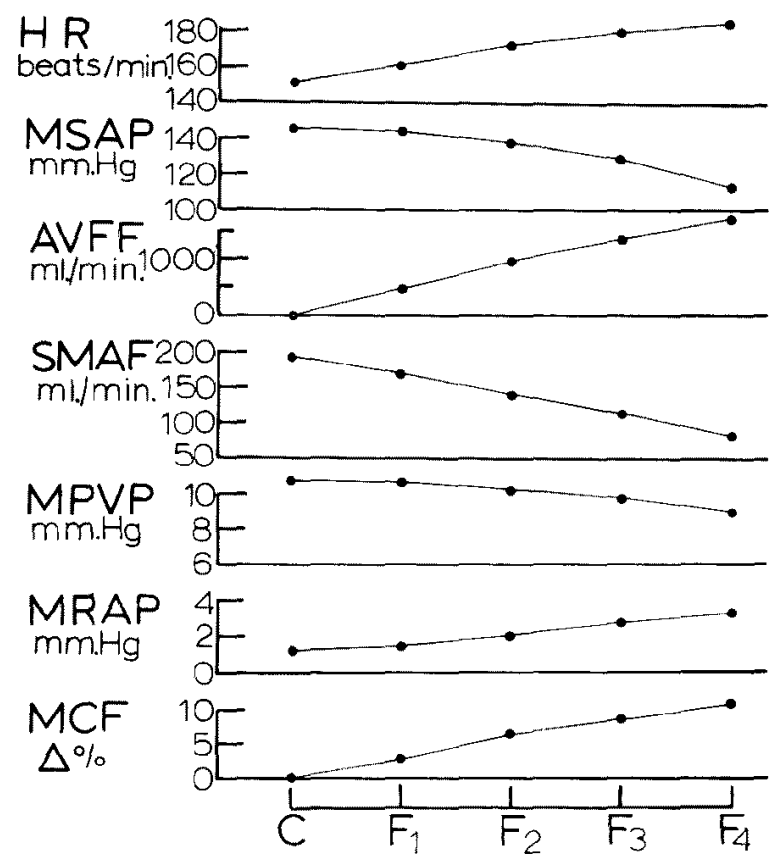

Fig. 5. The effects of A-V fistula on heart rate (HR), mean systemic arterial pressure (MSAP), A-V fistula blood flow (AVFF), superior mesenteric arterial blood flow (SMAF), mean portal venous pressure (MPVP), mean right atrial pressure (MRAP) and myocardial contractile force (MCF) in a dog. $G$ and $F_{1-4}$ denote values during control and during $A \cdot V$ fistulas of severity increasing.

tension (dp/dt) significantly. Upon cessation, all the hemodynamic parameters returned to control values within $3 \mathrm{~min}$.

3. Effect of A-V Fistula on the Splanchnic Circulation

The effects of A-V fistula on mean systemic arterial pressure, superior mesenteric arterial blood flow, portal venous pressure, right atrial pressure and myocardial contractile force were studied in 6 dogs. The results are qualitatively similar and consistent in all experiments. As seen in Fig. 5, A-V fistula caused increases in heart rate, portal venous pressure and peripheral resistance in the superior mesenteric artery, and decreases in mean systemic arterial pressure and superior mesenteric arterial blood flow.

4. Effect of A-V Fistula on the Femoral Circulation

The effects of unilateral femoral A-V fistula on the bilateral femoral arterial pressure, venous pressure and blood flows were studied in 6 dogs. The results were qualitatively similar and consistent. A tracing of the representative experiments is illustrated in Fig. 6. The left femoral A-V fistula decreased bilateral femoral arterial pressures and blood flows. The decreases in the 


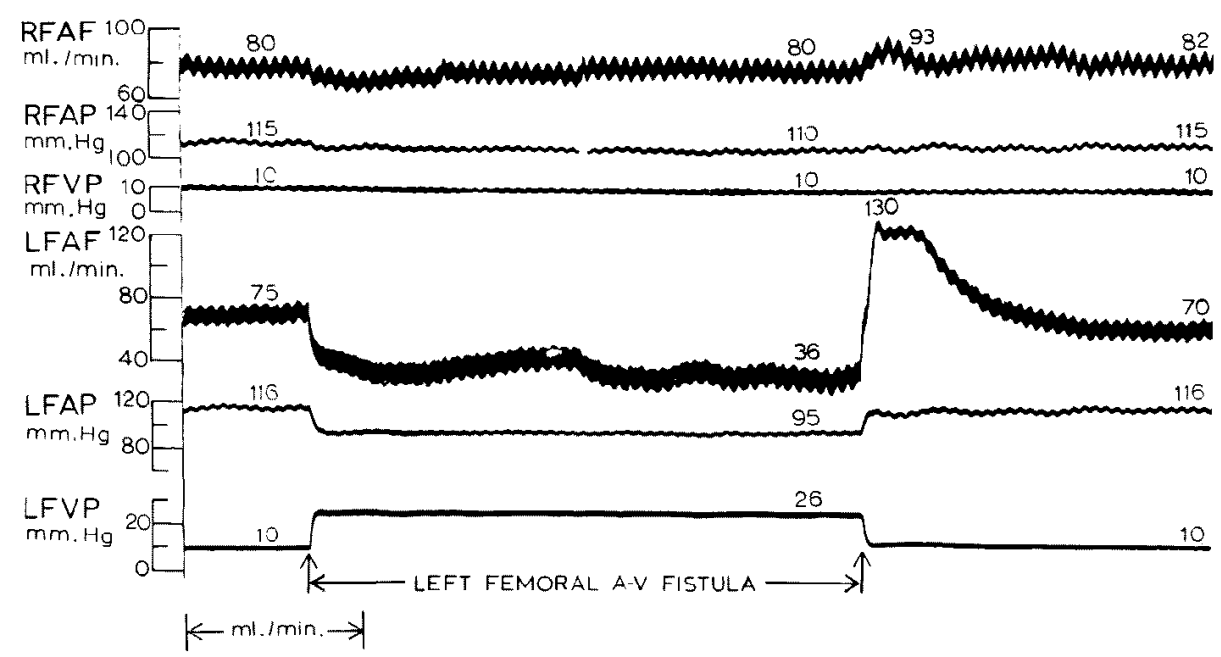

Fig. 6. The effects of a left femoral A-V fistula on right femoral arterial blood flow (FRAF), right femoral arterial pressure (RFAP), right femoral venous pressure (RFVP), left femoral arterial blood flow (LFAF), left femoral arterial pressure (LFAP), and left femoral venous pressure (LFVP) in a dog. $A$ left femoral A-V fistula was induced between 2 vertical arrows. Left femoral arterial blood flow, and left femoral arterial and venous pressures were measured at the sites peripheral to the A-V fistula.

ipsilateral femoral arterial pressure and flow were always greater than those in the contralateral. On the other hand, the femoral A-V fistula increased markedly the ipsilateral femoral venous pressure peripheral to the site of the A-V fistula but caused no significant change in the contralateral femoral venous pressure. The magnitude of the changes of the above mentioned hemodynamic parameters was essentially in proportion to the size of the A-V fistula produced.

\section{Discussion}

It is evident from the present study that the sudden opening of an $\mathrm{A}-\mathrm{V}$ fistula decreases total peripheral resistance, mean systemic arterial pressure, effective systemic blood flow, and increases total cardiac output, pulmonary arterial blood flow and systemic venous reiurn precipitously and concomitantly at its onset. The increase in total cardiac output results from a simultaneous increase in venous return and decrease in total periphcral resistance. This hemodynamic change appeared to be evidenced by the abrupt decrease and increase in the left and right ventricular end-diastolic pressures, respectively, as seen in Fig. 3. Stepwise increases in the severity of the A-V fistula cause stepwise increases in total cardiac output, but stepwise decrease in effective 
systemic blood flow. The increments in total cardiac output and fistula flow are greater than the decrement in effective systemic flow (Fig. 2). This increase in total cardiac output is essentially derived from the increased stroke volume, since the increment in stroke volume is far greater than that in heart rate. Furthermore, the increase in cardiac output following A-V fistula was observed without any change in heart rate. The previous studies from this laboratory and others have shown that the increase in heart rate of more than 120 beats/min. increased cardiac output very little or not at all and reduced stroke volume progressively. Tachycardias of rates more than 160 beats $/ \mathrm{min}$. usually reduce stroke volume, and cardiac output essentially in proportion to the rates. Hence, the increased stroke volume and cardiac output are most likely due to the increased systemic venous return and subsequently to the enhanced ventricular filling, and also to the increased myocardial contractility mediated through the operation of Frank-Starling's law of the heart. The previous studies in the isolated metabolically supported hearts, ${ }^{10)}$ showed that the increase in cardiac work by augmenting cardiac output per se was accompanied by a relatively slight increase in cardiac oxygen uptake. Therefore, in A-V fistula, the heart is capable of performing a great deal of work without decreasing cardiac efficiency. ${ }^{21}$

As shown in the present and previous studies from this laboratory, systemic arterial pressure and systemic blood flow fell suddenly and markedly upon the induction of $\mathrm{A}-\mathrm{V}$ fistula, and then gradually returned toward control level. The marked improvement of these parameters without any significant change in the $\mathrm{A}-\mathrm{V}$ fistula blood flow is most likely elicited by the increased activity of the sympathoadrenal system, ${ }^{4}$ since this hemodynamic improvement was absent or markedly reduced in reserpinized dogs (unpublished data). The increased sympathoadrenal activity was also evidenced by the increases in myocardial contractile force, $\mathrm{dp} / \mathrm{dt}$ values, heart rate and systemic peripheral resistance, and by the improvement of left ventricular function curve. It has been shown that the administration of catecholamines and the sympathetic nerve stimulation improved left myocardial contractility and left ventricular performance considerably. ${ }^{1)-31}$ From the results obtained from the present study, it appears obvious that the enhanced sympathoadrenal activity plays an important role on the cardiovascular regulation in acute A-V fistula, since it not only improves myocardial contractility and cardiac performance, but also increases systemic venous return by a decrease in the peripheral vascular capacitance. ${ }^{14)}$ Recently Chidsey and his associates ${ }^{15)}$ found that the myocardial contents of catecholamines are markedly reduced in the presence of congestive heart failure. It seems that the cardiovascular responses to the sympathoadrenal stimulation may be different in dogs with chronic A-V fistula 
from those with acute $\mathrm{A}-\mathrm{V}$ fistula.

\section{ACKNOWLEDGEMENT}

The author wished to acknowledge the able technical assistance of J. R. Mc Curdy, B. A. Darrow, Z. S. Williams, G. Mercer, and D. Hite, Sodium heparin solution (Panheparin and Liquaemin) were generously supplied by Dr. H.G. Schoepke of Abbott Laboratories, North Chicago, Ill., and Dr. H. A. Strade of Organon, Inc., West Orange, N. J.

This work was supported in part by research grants (HE 11848 and HE 08057) from the U. S. Public Health Service.

\section{REFERENCES}

1. Frank, G. W., Wang, H. H., Lammerant, J., Miller, R., and Wegria, R.: J. Clin. Invest, 34: $722,1955$.

2. Wegria, R., Nakano, J, McGiff, J. C., Rochester, D. F., Bluemthal, M. R., and Muraviev, T.: Am. J. Physiol. 193: 147, 1958.

3. Glaviano, V. V.: Am. J. Physiol. 197: 201, 1959.

4. Nakano, J., Zekert, H., Griege, C. W., Wang, K. M., Schaefer, H. S., and Wegria, R.: Am. J. Physiol. 202: 413, 1961 .

5. Guyton, A. C. and Sagawa, K.: Am. J. Physiol. 200: 1157, 1961.

6. Nakano, J. and De Schryver, C.: Am. J. Physiol. 207: 1319, 1965.

7. Siegel, J. H. and Sonnenblick, E. H.: Circulat. Res. 12: 1597, 1963.

8. Nakano, J.: J. Pharmacol. Exp. Therap. 157: 19, 1967.

9. Shipley, R. E. and Wilson, C.: Proc. Soc. Exp. Biol. Med. 78: 724, 1951.

10. Sarnoff, S. J., Braunwald, E., Welch, G. H., Case, R. B., Stainsby, W. N., and Macruz, R.: Am. J. Physiol. 192: 148, 1958.

11. Randall, W. C. and Rohse, W. G.: Circulat. Res. 4: 470, 1956.

12. Sarnoff, S. J., Brockman, S. K., Gilmore, J. P., Linden, R.J., and Mitchell, J. H.: Girculat. Res. 8 : 1108, 1960.

13. Nakano, J. and Kusakari, T.: Am. J. Physiol. 210: 833, 1966.

14. Mellander, S. and Lewis, D. H.: Circulat. Res. 8: 105, 1963.

15. Chidsey, C. A., Kaiser, G. A., Sonnenblick, E. H., Spann, J. F., Jr., and Braunwald, E.: J. Clin. Invest. 43 : 2386, 1964. 